\title{
Analytical Approach to Regularization Design for Isotropic Spatial Resolution
}

\author{
Jeffrey A. Fessler, Senior Member, IEEE
}

\begin{abstract}
In emission tomography, conventional quadratic regularization methods lead to nonuniform and anisotropic spatial resolution in penalized-likelihood (or MAP) reconstructed images, even for idealized shift-invariant imaging systems. Previous methods for designing regularizers that improve resolution uniformity have used matrix manipulations and discrete Fourier transforms. This paper describes a simpler approach for designing datadependent, shift-variant regularizers. We replace the usual discrete system models used in statistical image reconstruction with locally shift-invariant, continuous-space approximations, and design the regularizer using analytical Fourier transforms. We discretize the final analytical solution to compute the regularizer coefficients. This new approach requires even less computation than previous approaches that used FFTs, and provides additional insight into the problem of designing regularization methods to achieve uniform, isotropic spatial resolution.
\end{abstract}

Index Terms-PET, SPECT, penalized-likelihood image reconstruction, tomography, maximum a posteriori (MAP) estimation.

\section{INTRODUCTION}

When reconstructing PET and SPECT emission or transmission images by penalized-likelihood (or MAP) methods, interactions between the log-likelihood for Poisson measurements and conventional quadratic regularizers lead to nonuniform and anisotropic spatial resolution. This undesirable property holds even for idealized shift-invariant imaging systems [1]. As an illustration of the problematic effects of nonuniform resolution, the "conventional" regularization results in Fig. 2 show nonuniform apparent intensity around the rings. Using a quadratically-penalized, unweighted least-squares (QPULS) estimation method could circumvent this problem, but QPULS images have poor noise properties (akin to the filter-backproject (FBP) method). Statistical weighting, which is explicit in PWLS methods [2] and implicit in penalized-likelihood methods, is a central advantage of statistical methods over FBP.

Methods for designing regularizers to try to improve the uniformity and isotropy of the reconstructed images include [1], [3]-[5]. Methods for providing uniform pixel contrast have also been proposed [6]. Most of the more recent methods provide reasonable regularization designs. All previous methods have been based on the usual discrete system models associated with statistical image reconstruction. So all of these methods involve various system matrix manipulations. The matrix approach could be critiqued as lacking the type of insight

Supported in part by NSF grant BES-9982349 and NIH grant CA-60711. EECS Dept., The University of Michigan, fessler@umich.edu that is associated with analytical formulations. (Consider the importance of the concept of the $1 / r$ response associated with projection/backprojection, for example.) In this paper, we use analytical Fourier transforms to develop a simplified regularization design method, completing an idea first described in [7]. In essence, we solve the design optimization problem in continuous space, and then discretize the solution for implementation. The solution is a regularization method that is then used for penalized-likelihood image reconstruction with any of the available iterative algorithms.

\section{THEORY}

There are two possible formulations of new method. One approach formulates the entire problem using continuous-space operators (e.g., the Radon transform) and solves the problem in that domain, discretizing only at the very end. This formulation seems more "pure" but requires lengthier exposition. Instead, here we start with a discrete formulation, convert to continuousspace only at a critical step, solve that step in continuous space, and again discretize at the end.

We use two key concepts from previous work. One concept is local shift invariance [3], [8], [9]. Although emission tomography systems are shift variant, locally to any given pixel, the system and reconstruction method are often approximately shift invariant, allowing a "local Fourier analysis." The other concept is that for any given pixel, the effect of (implicit or explicit) ray-dependent weighting in a statistical reconstruction method can be approximated by one weight per projection angle [3].

\section{A. Local impulse response}

Let $\boldsymbol{y}$ denote the projection measurement vector, $\boldsymbol{A}$ the system matrix, and $\boldsymbol{x}$ the unknown object vector (pixel values), where $\boldsymbol{y} \approx \boldsymbol{A} \boldsymbol{x}$. For an estimator $\hat{\boldsymbol{x}}(\boldsymbol{y})$, we define the local impulse response for the $j$ th pixel to be

$$
\boldsymbol{l}^{j}=\lim _{\delta \rightarrow 0} \frac{\hat{\boldsymbol{x}}\left(\boldsymbol{y}+\delta \boldsymbol{A} \boldsymbol{e}_{j}\right)-\hat{\boldsymbol{x}}(\boldsymbol{y})}{\delta} .
$$

This PSF describes how a small impulse in the $j$ th pixel affects other pixels, and is our tool for regularization design.

\section{B. Regularized reconstruction}

Penalized likelihood reconstruction methods have the form

$$
\hat{\boldsymbol{x}}=\underset{\boldsymbol{x}}{\arg \min } L(\boldsymbol{A x}, \boldsymbol{y})+R(\boldsymbol{x}),
$$


where $L$ denotes the negative $\log$-likelihood and $R(\boldsymbol{x})$ denotes a roughness penalty function that regularizes the problem. We focus here on quadratic roughness penalties of the form $R(\boldsymbol{x})=$ $\frac{1}{2} \boldsymbol{x}^{\prime} \boldsymbol{R} \boldsymbol{x}$, where $\boldsymbol{R}$ is the Hessian of the penalty function.

For such estimators, the local impulse response is [1], [5]:

$$
\boldsymbol{l}^{j}=\left[\boldsymbol{A}^{\prime} \nabla^{20} L(\boldsymbol{A} \hat{\boldsymbol{x}}, \boldsymbol{y}) \boldsymbol{A}+\boldsymbol{R}\right]^{-1} \boldsymbol{A}^{\prime}\left[-\nabla^{11} L(\boldsymbol{A} \hat{\boldsymbol{x}}, \boldsymbol{y})\right] \boldsymbol{A} \boldsymbol{e}_{j} .
$$

For typical log-likelihoods, this simplifies as follows [5]:

$$
\boldsymbol{l}^{j}=\left[\boldsymbol{A}^{\prime} \boldsymbol{W} \boldsymbol{A}+\boldsymbol{R}\right]^{-1} \boldsymbol{A}^{\prime} \boldsymbol{W} \boldsymbol{A} \boldsymbol{e}_{j},
$$

where $\boldsymbol{W}$ is diagonal and depends on the log-likelihood and $\boldsymbol{y}$, e.g., for emission tomography $\boldsymbol{W} \approx \operatorname{diag}\left\{1 / y_{i}\right\}$.

For simplicity, we focus on regularization methods using first-order differences of the form

$$
R(\boldsymbol{x})=\sum_{n, m} \sum_{l=1}^{L} r_{l}^{j}\left(\left(c_{l} * * x\right)[n, m]\right)^{2},
$$

where * denotes 2D convolution and, with slight notation recycling, $x[n, m]$ denotes the $2 \mathrm{D}$ array corresponding to the lexicographically ordered vector $\boldsymbol{x}$, and

$$
c_{l}[n, m]=\frac{1}{\sqrt{n_{l}^{2}+m_{l}^{2}}}\left(\delta_{2}[n, m]-\delta_{2}\left[n-n_{l}, m-m_{l}\right]\right),
$$

where $\left\{\left(n_{l}, m_{l}\right)\right\}$ denote the offsets to the $j$ th pixel's neighbors, and $\delta_{2}[n, m]$ denotes the 2D Kronecker impulse. Above, $j$ is the lexicographic index corresponding to the pixel at $[n, m]$. The methods generalize readily to higher-order differences and to 3D problems. We focus on the case using the nearest horizontal, vertical, and diagonal neighbors ${ }^{1}$

$$
\begin{aligned}
c_{1}[n, m] & =\delta_{2}[n, m]-\delta_{2}[n-1, m-0] \\
c_{2}[n, m] & =\delta_{2}[n, m]-\delta_{2}[n-0, m-1] \\
c_{3}[n, m] & =\frac{1}{\sqrt{2}}\left(\delta_{2}[n, m]-\delta_{2}[n-1, m-1]\right) \\
c_{4}[n, m] & =\frac{1}{\sqrt{2}}\left(\delta_{2}[n, m]-\delta_{2}[n-1, m+1]\right) .
\end{aligned}
$$

\section{Regularization design}

Our goal is to choose the penalty coefficients $\boldsymbol{r}^{j}=$ $\left(r_{1}, \ldots, r_{L}\right)$ (and hence $\boldsymbol{R}$ ) so that the local impulse response at each pixel $j$ closely matches some target PSF $\boldsymbol{l}_{0}$. In principle one could attempt to match any desired target PSF, but experience has shown that reasonable target PSFs have the form

$$
\boldsymbol{l}_{0}^{j}=\left[\boldsymbol{G}^{\prime} \boldsymbol{G}+\boldsymbol{R}_{0}\right]^{-1} \boldsymbol{G}^{\prime} \boldsymbol{G} \boldsymbol{e}_{j},
$$

where $G$ denotes a shift-invariant system that approximates the possibly shift-varying system model $\boldsymbol{A}$, and $\boldsymbol{R}_{0}$ denotes

\footnotetext{
${ }^{1}$ Note that the diagonal differences are divided by $\sqrt{2}$, so the penalty includes terms of the form $\left(\frac{x[n, m]-x[n-1, m-1]}{\sqrt{2}}\right)^{2}$. This differs from the common practice [2], [4] of dividing the square of the diagonal differences by $\sqrt{2}$, i.e., $\frac{1}{\sqrt{2}}(x[n, m]-x[n-1, m-1])^{2}$. Our analysis suggests that the common practice is suboptimal for producing isotropic spatial resolution.
}

a conventional shift-invariant regularizer. In other words, we would like to choose $\boldsymbol{R}$ (by choosing $\left\{\boldsymbol{r}^{j}\right\}$ ) such that

$$
\left[\boldsymbol{A}^{\prime} \boldsymbol{W} \boldsymbol{A}+\boldsymbol{R}\right]^{-1} \boldsymbol{A}^{\prime} \boldsymbol{W} \boldsymbol{A} \boldsymbol{e}_{j} \approx\left[\boldsymbol{G}^{\prime} \boldsymbol{G}+\boldsymbol{R}_{0}\right]^{-1} \boldsymbol{G}^{\prime} \boldsymbol{G} \boldsymbol{e}_{j} .
$$

Achieving this goal should lead to nearly uniform and anisotropic spatial resolution. (If some other resolution criterion is desired, then one can choose alternative $\boldsymbol{l}_{0}$ 's.)

Motivated by continuous-space analogs not shown, we "cross multiply," rearrange the matrices, and simplify, yielding

$$
\boldsymbol{R}_{0} A^{\prime} \boldsymbol{W} A e_{j} \approx R G^{\prime} G e_{j} .
$$

(Since all circulant matrices commute, matrices that are locally shift invariant will commute approximately.) Roughly speaking then, we would like to solve

$$
\min _{\left\{\boldsymbol{r}^{j}\right\}} \sum_{j}\left\|\boldsymbol{R}_{0} \boldsymbol{A}^{\prime} \boldsymbol{W} \boldsymbol{A} \boldsymbol{e}_{j}-\boldsymbol{R} \boldsymbol{G}^{\prime} \boldsymbol{G} \boldsymbol{e}_{j}\right\|,
$$

where $\boldsymbol{R}$ depends on $\left\{\boldsymbol{r}^{j}\right\}$, for some type of norm. Previous methods have used matrices and FFTs at this point [3], [5].

\section{Analytical formulation}

We propose to replace each of the four matrices in (4) with a corresponding continuous-space convolution operator, expressed using its Fourier transform. For each operator we consider the "local perspective" of the $j$ th pixel.

The simplest term is $\boldsymbol{G}^{\prime} \boldsymbol{G}$. If we replaced $\boldsymbol{G}$ with the ideal Radon transform, then $G^{\prime} G$ would be the combination of forward and back-projection, corresponding to convolution with $1 / r$ in continuous-space, or multiplication by $1 / \rho$ in the Fourier domain, where $(\rho, \varphi)$ denote polar coordinates in frequency space. A more reasonable model would account at least for some "typical" detector response, so we use

$$
\boldsymbol{G}^{\prime} \boldsymbol{G} \equiv \frac{|B(\rho)|^{2}}{\rho},
$$

where $B(\cdot)$ denotes some user-selected frequency response, perhaps preferably corresponding to the "typical" radial blur function, e.g., the blur at the center of a single projection view. However, the details of $B$ turn out not to be critical; in fact, we require only that $B(\rho)>0$. The idea here is to replace the matrix $\boldsymbol{G}$ with the continuous-space operator $\mathcal{G}=\mathcal{B} \mathcal{P}$, where $\mathcal{B}$ denotes view-independent, radially shiftinvariance blur, and $\mathcal{P}$ denotes the usual Radon transform. Note that $\mathcal{G}^{*} \mathcal{G}=\mathcal{P}^{*} \mathcal{B}^{*} \mathcal{B} \mathcal{P}$, where $\mathcal{P}^{*}$ is the adjoint of $\mathcal{P}$ (i.e., backprojection). $\mathcal{B}$ appears twice, causing the "square" in (5).

For shift varying systems, let $b_{\varphi}^{j}(r)$ denote the detector response at angle $\varphi$ local to where the $j$ th pixel projects onto the detector at that angle. (For a shift-invariant system $b(r)$ would be independent of $\varphi$ and $j$.) Let $B_{\varphi}^{j}(\rho)$ denote the corresponding (local) frequency response. Similarly, let $w_{\varphi}(r)$ denote the diagonal "element" of $\boldsymbol{W}$ corresponding to angle $\varphi$ and radial position $r$, and define the following effective "certainty" at angle $\varphi$ for the $j$ th pixel (cf. [3, eq. (19)]):

$$
w^{j}(\varphi)=\frac{\int_{-\infty}^{\infty}\left|b_{\varphi}^{j}(r)\right|^{2} w_{\varphi}(r) \mathrm{d} r}{\int_{-\infty}^{\infty}\left|b_{\varphi}^{j}(r)\right|^{2} \mathrm{~d} r} .
$$


Using continuous-space analogies, one can show

$$
\boldsymbol{A}^{\prime} \boldsymbol{W} \boldsymbol{A} \equiv \frac{w^{j}(\varphi)\left|B_{\varphi}^{j}(\rho)\right|^{2}}{\rho} .
$$

For continuous-space regularization of a $2 \mathrm{D}$ object $f$, the usual roughness penalty would be $R_{0}(f)=\int\|\nabla f\|^{2}$. The derivative property of the Fourier transform leads to

$$
\boldsymbol{R}_{0} \equiv|2 \pi \rho|^{2} .
$$

Finally, analysis of the quadratic penalty function (1) leads to

$$
\boldsymbol{R} \equiv \sum_{l=1}^{L} r_{l}^{j}|2 \pi \rho|^{2} \cos ^{2}\left(\varphi-\varphi_{l}\right),
$$

where $\varphi_{l}=\tan ^{-1} \frac{m_{l}}{n_{l}}$ and $\left(n_{l}, m_{l}\right)$ denote the offsets in (2).

Substituting these four continuous-space approximations into (4) leads to the minimization problem

$$
\begin{gathered}
\boldsymbol{r}^{j}=\underset{\boldsymbol{r} \succeq \mathbf{0}}{\arg \min } \int_{0}^{\pi} \int_{-\infty}^{\infty} \frac{v(\rho)}{2 \pi} \\
\left(w^{j}(\varphi)\left|B_{\varphi}^{j}(\rho)\right|^{2}-|B(\rho)|^{2} \sum_{l=1}^{L} r_{l} \cos ^{2}\left(\varphi-\varphi_{l}\right)\right)^{2} \mathrm{~d} \rho \mathrm{d} \varphi
\end{gathered}
$$

where $v(\rho)$ is a user-selected weighting function. The nonnegativity constraint ensures that $\boldsymbol{R}$ is nonnegative definite. Expanding the quadratic, integrating over $\rho$, and completing the square leads to the following simplified expression:

$$
\hat{\boldsymbol{r}}^{j}=\underset{\boldsymbol{r} \succeq \mathbf{0}}{\arg \min } \int_{0}^{\pi}\left(\bar{w}^{j}(\varphi)-\sum_{l=1}^{L} r_{l} \cos ^{2}\left(\varphi-\varphi_{l}\right)\right)^{2} \mathrm{~d} \varphi,
$$

where

$$
\bar{w}^{j}(\varphi)=w^{j}(\varphi) \frac{\int_{-\infty}^{\infty} v(\rho)|B(\rho)|^{2}\left|B_{\varphi}^{j}(\rho)\right|^{2} \mathrm{~d} \rho}{\int_{-\infty}^{\infty} v(\rho)|B(\rho)|^{4} \mathrm{~d} \rho} .
$$

For the "convenient" choice $v(\rho)=1 /|B(\rho)|^{2}$, it follows from Parseval's theorem that $\bar{w}^{j}(\varphi)$ is

$$
w^{j}(\varphi) \frac{\int_{-\infty}^{\infty}\left|B_{\varphi}^{j}(\rho)\right|^{2} \mathrm{~d} \rho}{\int_{-\infty}^{\infty}|B(\rho)|^{2} \mathrm{~d} \rho}=w^{j}(\varphi) \frac{\int_{-\infty}^{\infty}\left|b_{\varphi}^{j}(r)\right|^{2} \mathrm{~d} r}{\int_{-\infty}^{\infty}|b(r)|^{2} \mathrm{~d} r} .
$$

The denominator is a constant that can be absorbed into $\beta$. Interestingly, for this choice of weighting $v(\rho)$, the choice of $B(\cdot)>0$ has no influence on the design of $\boldsymbol{R}$.

Combining (9) with (6) yields

$$
\bar{w}^{j}(\varphi)=\frac{\int\left|b_{\varphi}^{j}(r)\right|^{2} w_{\varphi}(r) \mathrm{d} r}{\int|b(r)|^{2} \mathrm{~d} r} .
$$

For implementation, we ignore the denominator since it is independent of $\varphi$ and $j$, and compute

$$
\bar{w}^{j}(\varphi)=\sum_{i \in \mathcal{I}_{\varphi}} a_{i j}^{2} w_{i},
$$

where $\boldsymbol{A}=\left\{a_{i j}\right\}$ and $\mathcal{I}_{\varphi}$ denotes the set of rays that correspond to the projection at angle $\varphi$. This expression is remarkably similar to the weighting proposed in [4, eqn. (33)] using a quite different derivation.

\section{E. Analytical solution}

Now to solve the minimization (8). Expanding the cosines in terms of a 3-term basis that is orthonormal with respect to the inner product $\langle f, g\rangle=\frac{1}{\pi} \int_{0}^{\pi} f(\varphi) g(\varphi) \mathrm{d} \varphi$ yields

$$
\begin{aligned}
\cos ^{2}\left(\varphi-\varphi_{l}\right)= & \frac{1}{2} \cdot 1+\frac{\cos \left(2 \varphi_{l}\right)}{2 \sqrt{2}}[\sqrt{2} \cos (2 \varphi)] \\
& +\frac{\sin \left(2 \varphi_{l}\right)}{2 \sqrt{2}}[\sqrt{2} \sin (2 \varphi)] .
\end{aligned}
$$

Using the second-order neighborhood (3), we find $\varphi_{1}=$ $0, \varphi_{2}=\pi / 2, \varphi_{3}=\pi / 4, \varphi_{4}=-\pi / 4$, so the minimization problem (8) simplifies to

$$
\hat{\boldsymbol{r}}^{j}=\underset{\boldsymbol{r} \succeq \mathbf{0}}{\arg \min } \Psi(\boldsymbol{r}), \quad \Psi(\boldsymbol{r})=\frac{1}{2}\|\boldsymbol{T r}-\boldsymbol{b}\|^{2},
$$

for each $j$, where $\boldsymbol{T}$ is the $3 \times 4$ matrix (for $L=4$ ):

$$
\begin{gathered}
\boldsymbol{T}=\frac{1}{2}\left[\begin{array}{cccc}
1 & 1 & 1 & 1 \\
1 / \sqrt{2} & -1 / \sqrt{2} & 0 & 0 \\
0 & 0 & 1 / \sqrt{2} & -1 / \sqrt{2}
\end{array}\right] \\
\boldsymbol{b}=\left[\begin{array}{c}
d_{1} \\
\sqrt{2} d_{2} \\
\sqrt{2} d_{3}
\end{array}\right], \boldsymbol{d}=\left[\begin{array}{l}
\frac{1}{\pi} \int_{0}^{\pi} \bar{w}^{j}(\varphi) \mathrm{d} \varphi \\
\frac{1}{\pi} \int_{0}^{\pi} \bar{w}^{j}(\varphi) \cos (2 \varphi) \mathrm{d} \varphi \\
\frac{1}{\pi} \int_{0}^{\pi} \bar{w}^{j}(\varphi) \sin (2 \varphi) \mathrm{d} \varphi
\end{array}\right] .
\end{gathered}
$$

When $w_{\varphi}(r)$ is the inverse of the variance of the projection data at $(r, \varphi)$, one can think of $d_{1}$ as quantifying the overall "certainty," $d_{2}$ is related to the horizontal and vertical directions, and $d_{3}$ is related to the diagonal directions.

This is an under-determined system, which is somewhat intuitive since one can obtain approximately isotropic smoothing using only the horizontal and vertical neighbors, or only the diagonal neighbors. For the purposes of regularization design, an under-determined situation is desirable since it allows us to use the "extra" degrees of freedom to ensure nonnegativity even when anisotropic regularization is needed.

We could solve the minimization (13) using the iterative NNLS algorithm [10, p. 158]. However, using the properties of $\boldsymbol{T}$ and $\boldsymbol{d}$, we can avoid iterations entirely by solving (12) analytically using the KKT conditions.

It follows from (13) that $\sqrt{d_{2}^{2}+d_{3}^{2}} \leq d_{1}$. The structure of $\boldsymbol{T}$ leads to eight-fold symmetry that simplifies analysis. If $d_{2}<0$ we can solve for $\boldsymbol{r}$ using $\left|d_{2}\right|$ and then swap $r_{1}$ with $r_{2}$. If $d_{3}<0$ we can solve for $\boldsymbol{r}$ using $\left|d_{3}\right|$ and then swap $r_{3}$ with $r_{4}$. If $d_{3}>d_{2}$ we can solve for $\boldsymbol{r}$ with $d_{2}$ and $d_{3}$ interchanged, and then swap $r_{1}$ with $r_{3}$ and $r_{2}$ with $r_{4}$. Therefore, hereafter we focus on cases where $0 \leq d_{3} \leq d_{2} \leq d_{1}$. Fig. 1 shows these first octant cases, numbered according to the number of nonzero elements of $\boldsymbol{r}$.

1 If $d_{2} \geq \frac{1}{2} d_{1}$ and $d_{3} \leq \frac{2}{3} d_{2}-\frac{1}{3} d_{1}$, then $r_{1}=\frac{4}{3}\left(d_{1}+d_{2}\right), \quad r_{2}=r_{3}=r_{4}=0$.

2 If $d_{3} \geq \frac{2}{3} d_{2}-\frac{1}{3} d_{1}$ and $d_{3}+d_{2} \geq \frac{1}{2} d_{1}$, then $r_{1}=\frac{8}{5}\left[\frac{1}{2} d_{1}+\frac{3}{2} d_{2}-d_{3}\right], \quad r_{2}=r_{4}=0$, $r_{3}=\frac{12}{5}\left[d_{3}-\left(\frac{2}{3} d_{2}-\frac{1}{3} d_{1}\right)\right]$.

3 If $d_{3}+d_{2} \leq \frac{1}{2} d_{1}$ and $d_{2} \geq \frac{1}{4} d_{1}$, then there are multiple nonnegative $\boldsymbol{r}$ that exactly solve $\nabla \Psi(\boldsymbol{r})=\mathbf{0}$. 


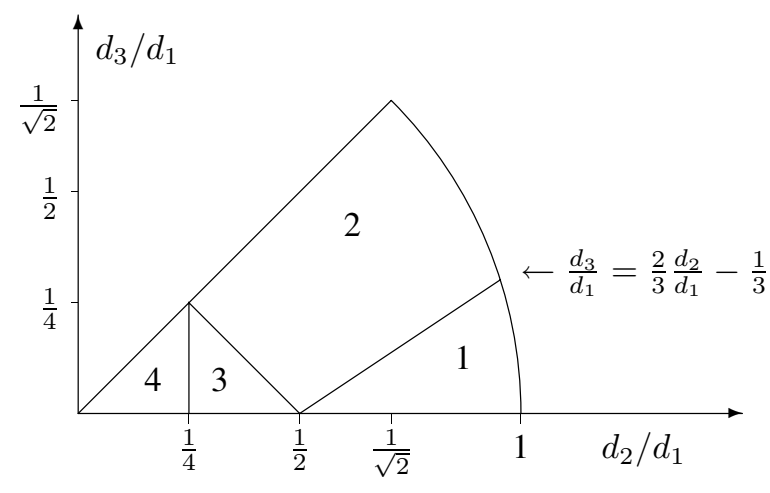

Fig. 1. First octant of quadratic penalty design space showing the four regions where different constraints are active.

The minimum-norm solution is $r_{1}=4 d_{2}, \quad r_{2}=0$, $r_{3}=d_{1}-2 d_{2}+2 d_{3}, \quad r_{4}=2\left[\frac{1}{2} d_{1}-\left(d_{2}+d_{3}\right)\right]$.

4 If $d_{2} \leq \frac{1}{4} d_{1}$, then there are multiple nonnegative $r$ that are exact solutions. The natural choice is the minimumnorm $r$ given by the pseudo-inverse solution $r=T^{\dagger} b$, where $r_{1}=2\left(\frac{1}{4} d_{1}+d_{2}\right), \quad r_{2}=2\left(\frac{1}{4} d_{1}-d_{2}\right)$, $r_{3}=2\left(\frac{1}{4} d_{1}+d_{3}\right), \quad r_{4}=2\left(\frac{1}{4} d_{1}-d_{3}\right)$.

The analytical solution presented above is for the usual firstorder differences with the second-order neighborhood (3). For higher-order differences or neighborhoods, it would appear to become increasingly cumbersome to solve (7) analytically, so the iterative NNLS approach may be more appealing. This can still be practical since $T$ is quite small.

The analytical solution above is a continuous function of $\boldsymbol{d}$, which in turn is a continuous function of $\bar{w}(\varphi)$. This continuity property would seem to be desirable for avoiding artifacts in the reconstructed images. For practical implementation, we simply discretize the integrals in (9) and (13) and use (11).

Matlab software for this design method is available in the tomography toolbox from:

http://www. eecs. umich.edu/ fessler/code.

\section{Simulation Results}

We have implemented the method both for the case $L=2$ (only horizontal and vertical neighbors) and the case $L=4$. We focus here on $L=4$ which gave better results. For simplicity we used quadratically-penalized weighted least squares (QPWLS) reconstruction rather than penalized likelihood, minimized by a preconditioned conjugate gradient (PCG) algorithm. The true image shown in Fig. 2, from [4], is $100 \times 100$ with $4 \mathrm{~mm}$ pixels. The sinogram was 102 bins by 80 views based on a shift-invariant system model with $4 \mathrm{~mm}$ ray spacing and strip width. To focus on resolution effects, no noise was added.

Fig. 2 shows the FBP result, which has uniform spatial resolution, and QPWLS with four different regularization methods: 1) conventional regularization, which has quite non-uniform and anisotropic resolution, 2) the "certainty-based" regularization from [1], which only corrects for average resolution
True Phantom

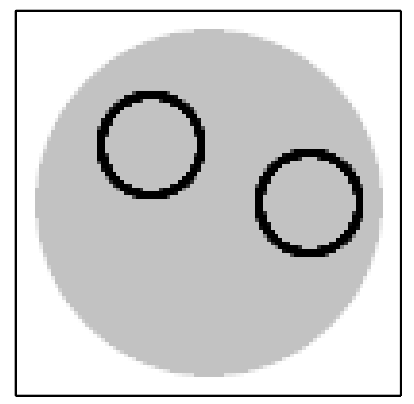

FBP

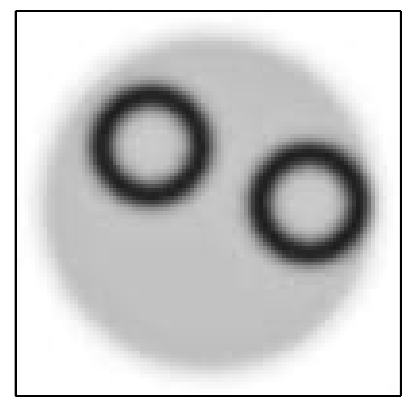

Conventional

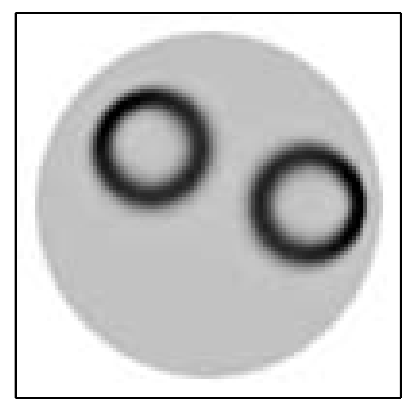

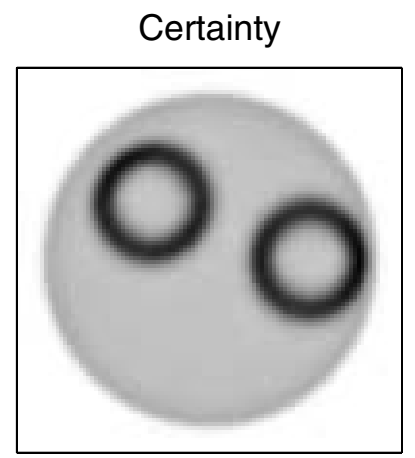

Nuyts

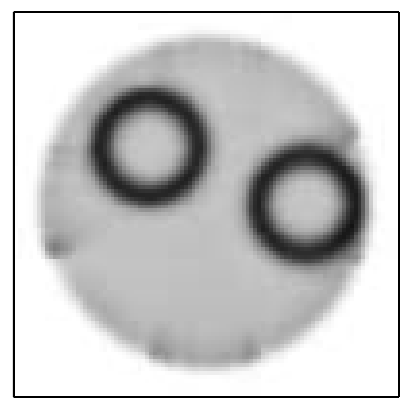

Proposed

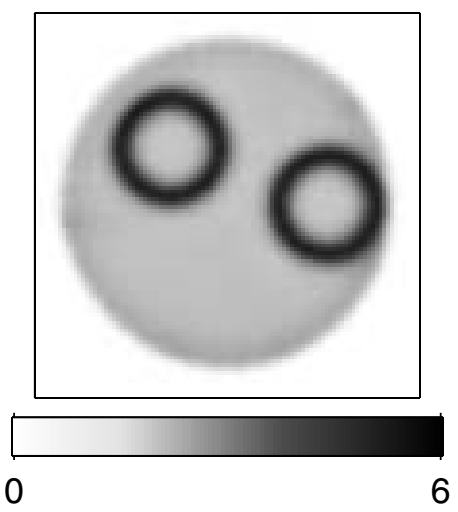

Fig. 2. True image and various noiseless reconstructions.

and not anisotropy, 3) the method proposed in [4], which works well except for some artifacts around the edges, and 4) the proposed analytical design method. Fig. 3 shows the calculated penalty coefficients $\left\{r_{l}^{j}\right\}$ for the method of [4] and the proposed method. Qualitatively they are fairly similar, although the differences do affect resolution properties.

Fig. 4 shows profiles around the rings verifying that the proposed design indeed improves uniformity relative to conventional regularization.

Using the methods of [1] we evaluated the local impulse response (PSF) at various spatial locations. The results for all methods do depend somewhat on spatial location. As an illustrative example, Fig. 5 shows the PSFs and their contours at one pixel. The proposed approach leads to more isotropic PSFs. 


\section{Discussion}

We have described a simple method for designing regularizers that lead to uniform and isotropic spatial resolution. No FFTs are required for implementation. The integrals in (13) are essentially three backprojections, but these can be simple backprojectors, perhaps even nearest-neighbor. Probably substantial angular down-sampling would be reasonable [5]. The method in [1] requires only one such backprojection, but does not correct for anisotropy. The other two backprojections give the necessary orientation information. The method of [4] uses four partial backprojections, each over $45^{\circ}$ of angles.

For $L=4$ we have a complete analytical solution. For $L>4$, we can use the efficient NNLS algorithm described in [3]. This extends the method to 3D systems and shift-variant problems.

The proposed solution provides new insight from recognizing that the least-squares problem (8) corresponds simply to fitting the angular weighting $\bar{w}^{j}(\varphi)$ using a small set of raised sinusoids with nonnegative coefficients. For example, for horizontal neighbors, the corresponding term is $\cos ^{2}(\varphi)=\frac{1}{2}+\frac{1}{2} \cos 2 \varphi$. This function peaks at $0^{\circ}$, which is logical since the horizontal penalty should be most affected by the $0^{\circ}$ projections (vertical rays). Likewise for the other neighboring pixel pairs.

The approach may also be useful for analysis of variance and covariance properties of regularized reconstruction methods.

\section{ACKNOWLEDGMENT}

The author gratefully acknowledges numerous enjoyable discussions with Web Stayman and Johan Nuyts.

\section{REFERENCES}

[1] J. A. Fessler and W. L. Rogers, "Spatial resolution properties of penalizedlikelihood image reconstruction methods: Space-invariant tomographs," IEEE Tr. Im. Proc., vol. 5, no. 9, pp. 1346-58, Sept. 1996.

[2] J. A. Fessler, "Penalized weighted least-squares image reconstruction for positron emission tomography," IEEE Tr. Med. Im., vol. 13, no. 2, pp. 290-300, June 1994

[3] J. W. Stayman and J. A. Fessler, "Regularization for uniform spatial resolution properties in penalized-likelihood image reconstruction," IEEE Tr. Med. Im., vol. 19, no. 6, pp. 601-15, June 2000.

[4] J. Nuyts and J. A. Fessler, "A penalized-likelihood image reconstruction method for emission tomography, compared to post-smoothed maximumlikelihood with matched spatial resolution," IEEE Tr. Med. Im., vol. 22, no. 9, pp. 1042-52, Sept. 2003.

[5] J. W. Stayman and J. A. Fessler, "Compensation for nonuniform resolution using penalized-likelihood reconstruction in space-variant imaging systems," IEEE Tr. Med. Im., 2003, to appear.

[6] J. Qi and R. M. Leahy, "Resolution and noise properties of MAP reconstruction for fully 3D PET," in Proc. of the 1999 Intl. Mtg. on Fully 3D Im. Recon. in Rad. Nuc. Med., 1999, pp. 35-9.

[7] J. A. Fessler, "Spatial resolution properties of penalized weighted least-squares image reconstruction with model mismatch," Comm. and Sign. Proc. Lab., Dept. of EECS, Univ. of Michigan, Ann Arbor, MI, 48109-2122, Tech. Rep. 308, Mar. 1997, Available from http://www. eecs. umich. edu/ fessler.

[8] J. A. Fessler and S. D. Booth, "Conjugate-gradient preconditioning methods for shift-variant PET image reconstruction," IEEE Tr. Im. Proc., vol. 8, no. 5, pp. 688-99, May 1999.

[9] J. Qi and R. M. Leahy, "A theoretical study of the contrast recovery and variance of MAP reconstructions with applications to the selection of smoothing parameters," IEEE Tr. Med. Im., vol. 18, no. 4, pp. 293-305, Apr. 1999.

[10] C. L. Lawson and R. J. Hanson, Solving least squares problems. PrenticeHall, 1974.
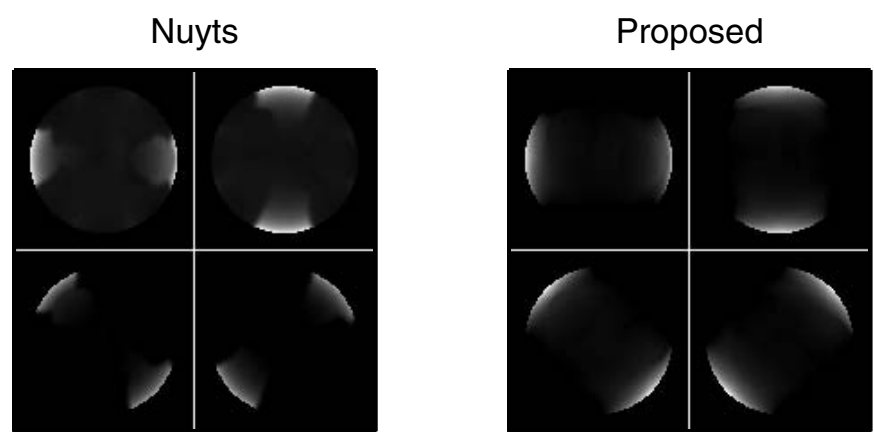

Fig. 3. Penalty coefficients $\left\{r_{l}^{j}\right\}_{l=1}^{4}$ for the method of [4] and the proposed method.

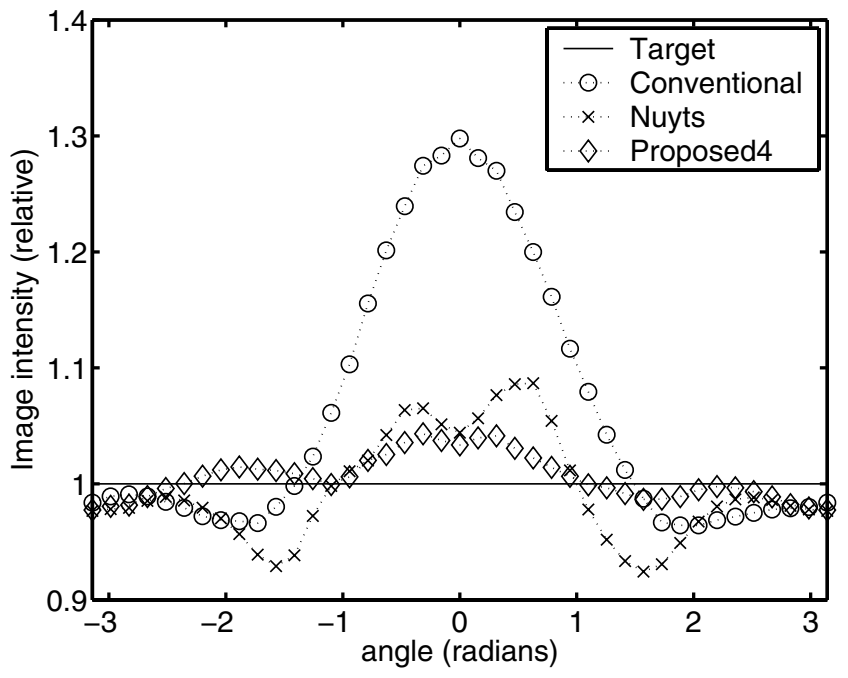

Fig. 4. Profile around right ring.

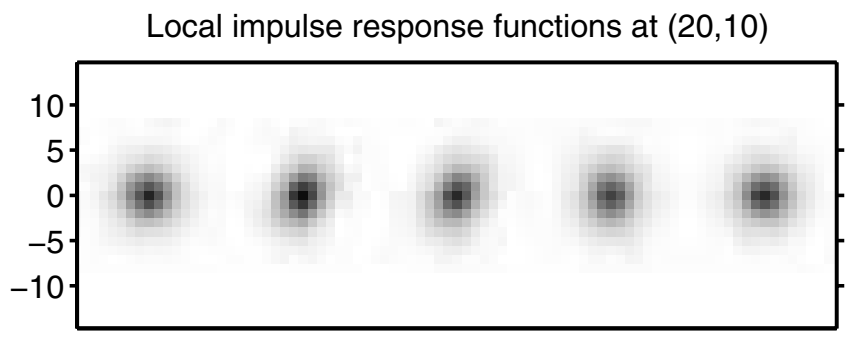

Contours

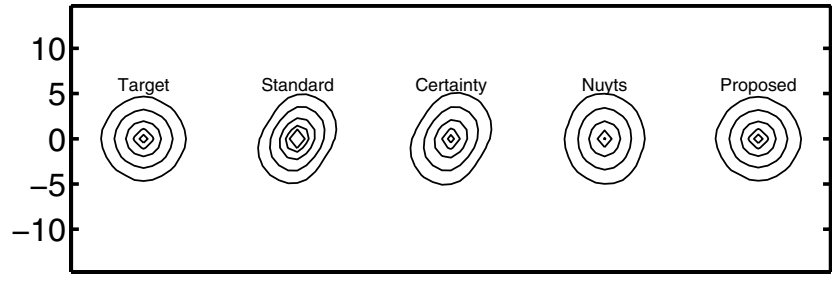

Fig. 5. PSF at $(20,0)$ for various regularization methods. 